\title{
The age structure of globular cluster systems in early-type galaxies and its dependence on host galaxy properties ${ }^{\star}$
}

\author{
M. Hempel ${ }^{1,2}$, M. Kissler-Patig ${ }^{3}$, T. H. Puzia ${ }^{4}$, and M. Hilker ${ }^{3,5}$
}

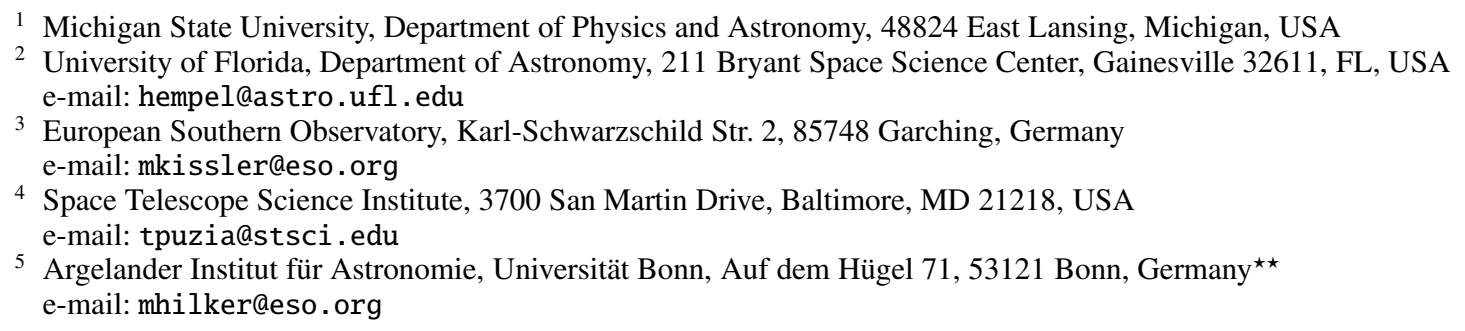

Received 25 July 2006 / Accepted 13 October 2006

ABSTRACT

\begin{abstract}
Aims. We present the results of an optical - near-infrared photometric survey of globular cluster systems in early-type galaxies. Our project aims to study correlations between the age structure of the globular cluster systems and environmental as well as structural parameters of their host galaxies.

Methods. To derive the age structure in globular cluster systems we use combined optical and near-infrared photometry. This combination facilitates the detection of young globular cluster sub-populations if their age differs significantly from the old ( $\sim 13 \mathrm{Gyr})$ population, i.e. if their age is $\$ 7 \mathrm{Gyr}$. We investigate globular cluster systems in galaxies located in the central regions of galaxy clusters (NGC 1399, M 87, NGC 4478), galaxies which are members of (sub-) groups (NGC 4365, NGC 5846) and isolated galaxies (NGC 3115 and NGC 7192). Further, we look for correlations between globular cluster system properties and structural parameters of the galaxies (velocity dispersion $\sigma$, color $\left((V-K),(B-V)\right.$ ), and luminosity $\left.M_{V}\right)$. For a quantitative analysis we define the "Methuselah" parameter, Mth - based on the fraction of young and old metal-rich globular clusters weighted by age, characterizing the age structure of a globular cluster system.

Results. We find that, within our galaxy sample, group galaxies appear to host an intermediate-age globular cluster population, whereas the globular cluster systems in cluster galaxies and in rather isolated galaxies are dominated by an old globular cluster population. A weak correlation of Mth with the $\mathrm{H}_{\beta}$ index of the integrated light of the host galaxies is the only trend found with respect to internal galaxy parameters.
\end{abstract}

Key words. galaxies: star clusters - galaxies: formation - galaxies: evolution - galaxies: fundamental parameters

\section{Tracing the star formation episodes in early-type galaxies}

The formation, evolution and star formation history of galaxies remains one of the most active fields in observational astronomy. Based on various photometric and spectroscopic studies of earlytype galaxies (and their globular cluster systems) our picture of these galaxies as an old, uniform, and rather predictable class of objects has changed significantly.

Various features of early-type galaxies are well explained by the commonly accepted formation scenarios, whereas others are in complete contradiction to it (e.g. Pahre 1999a). The fundamental plane (e.g. Djorgovski \& Davis 1987; Dressler et al. 1987), for instance, proved to be compatible over a long look-back time with an early major star-formation epoch. Overall, early-type galaxies appear to form a very homogeneous

* Based on observations at the Very Large Telescope of the European Southern Observatory, Chile (Program 63.N-0287, 64.N-0102).

$\star \star$ Founded by merging of the Sternwarte, Radioastronomisches Institut and Institut für Astrophysik und Extraterrestrische Forschung der Universität Bonn. population, consisting of an old stellar population and being similar in their basic features (e.g. see Bender \& Renzini 2005, and contributions therein). However, detailed spectroscopic investigations of the integrated light of nearby galaxies revealed a variety of structural features (e.g. Gorgas et al. 1990; Bender \& Surma 1992; Bender et al. 1992; de Zeeuw et al. 2002, the SAURON survey and references therein), that were neither expected nor visible in photometric studies. Counter- or co-rotating cores as well as massive dust shells turned out to be rather common amongst early-type galaxies and are clearly a sign of a more active evolution of these objects. An open question is whether these morphological signatures of a turbulent past also imply a significant perturbation of the star formation history of the host galaxy. In order to answer this question, sub-populations of different ages must be detected in the host galaxy, which proved to be very difficult by using the integrated light only. In contrast, identifying these sub-populations in the globular cluster systems appears to be a more promising route.

By representing single stellar populations (SSPs) and as a sign of the major star formation episodes, globular clusters are ideal probes of the evolutionary history of the parent galaxy. In order to assemble statistically relevant galaxy samples, 
Table 1. Relevant properties of our sample galaxies. The information was taken from: (1) de Vaucouleurs et al. (1991); (2) Tully \& Fisher (1988); (3) NED Data base and references therein; (4) Pahre (1999b); (5) Beuing et al. (2002).

\begin{tabular}{|c|c|c|c|c|c|c|c|c|}
\hline Property & NGC 1399 & NGC 1426 & NGC 3115 & NGC 4478 & NGC 4365 & M 87 & NGC 5846 & NGC 7192 \\
\hline Location & Fornax (cD) & Fornax (c) & Leo $(\mathrm{g})$ & Virgo & Virgo (c) & Virgo (cD) & Virgo-Libra & (g) \\
\hline$\rho\left[\mathrm{Mpc}^{-3}\right](2)$ & 1.59 & 0.66 & 0.08 & 3.92 & 2.93 & 4.17 & 0.84 & 0.28 \\
\hline$m-M(1)$ & 31.14 & 31.13 & 29.12 & 31.13 & 31.13 & 31.13 & 32.27 & 32.76 \\
\hline Type (1) & E1 & E4 & So & E2 & E3 & $\mathrm{E}+0-1, \mathrm{pec}$ & E0-1 & SA0 \\
\hline$B_{T, 0}(1)$ & 10.44 & 12.26 & 9.74 & 12.21 & 10.49 & 9.49 & 10.91 & 12.19 \\
\hline$(B-V)(2,3)$ & 1.26 & 0.89 & 1.04 & 0.60 & 1.05 & 0.86 & 1.07 & 0.97 \\
\hline$(V-K)_{0}(3)$ & 3.28 & 2.71 & 3.02 & 3.09 & 2.92 & 2.82 & 3.12 & 2.64 \\
\hline$M_{V}(2,3)$ & -21.55 & -19.74 & -20.22 & -19.78 & -21.57 & -22.50 & -22.23 & -21.52 \\
\hline$\sigma\left[\mathrm{km} \mathrm{s}^{-1}\right](2)$ & 308 & 155 & 264 & 143 & 261 & 333 & 252 & 184 \\
\hline $\mathrm{H}_{\beta}[\AA](5)$ & 1.19 & - & 1.85 & 1.84 & 1.73 & 1.07 & 2.02 & 1.51 \\
\hline$\left(\Delta \mathrm{H}_{\beta}\right)[\AA](5)$ & $( \pm 0.16)$ & - & $( \pm 0.11)$ & $( \pm 0.06)$ & $( \pm 0.12)$ & $( \pm 0.25)$ & $( \pm 0.09)$ & $( \pm 0.09)$ \\
\hline$D_{25}(4)$ & 3.3 & 2.2 & 6.1 & 1.9 & 6.2 & 9.2 & 4.9 & 1.7 \\
\hline \multirow[t]{2}{*}{ Peculiarities } & radio jet & - & - & - & KDC & radio & dust & $\mathrm{KDC}$ \\
\hline & - & - & - & - & - & X-ray & X-ray & - \\
\hline
\end{tabular}

Abbreviations: KDC- kinematically distinct core; c- galaxy cluster; g- galaxy group; cD- central cluster galaxy.

representing a specific set of galaxy parameters (e.g. environment, luminosity, ...), efficient photometric studies are used. Once the existence of age sub-populations within the globular cluster system has been established photometrically, more accurate ages for sub-sets of globular clusters can be determined using spectroscopy (e.g. Puzia et al. 2005).

The combination of optical and near-infrared photometry of globular clusters (e.g. Goudfrooij et al. 2001; Kissler-Patig et al. 2002; Puzia et al. 2002; Hempel \& Kissler-Patig 2004) has been introduced in order to reduce the degeneracy of age and metallicity effects and to allow the resolution of globular cluster age sub-populations. With the availability of infrared imagers on 8-10 m class telescopes, such as ISAAC (Infrared Spectrometer And Array Camera) at the Very Large Telescope (VLT) or space based instruments (e.g. NICMOS on board Hubble Space Telescope or IRAC on board the Spitzer Space Telescope) we are now able to complement, for a large galaxy sample, the vast number of optical data sets with infrared data. Here we present results of our ongoing study on early-type galaxies, which includes 8 galaxies, varying in parameters such as environment (represented by the Tully density parameter $\rho$ ), luminosity, velocity dispersion, and color. Basic information on the galaxy sample is given in Table 1.

\section{The age structure of a globular cluster system}

Our data and the analysis method are briefly detailed below.

\subsection{Outline of the method}

Optical and near-infrared photometric data of globular cluster systems are used to derive their cumulative age distribution. This is followed by the comparison with simulated systems of known age structure to constrain the later within the observed systems.

The goal is not to derive ages for individual globular clusters nor to determine absolute ages, both are currently out of reach given the photometric errors and model uncertainties. Instead we aim at the detection, among the metal-rich globular clusters, of age sub-populations differing by several Gyr. This age resolution is rather coarse, but sufficient to identify major star formation episodes that occurred at redshifts e.g. $<0.5, \sim 1$, or $>2$. This accuracy is useful to significantly constrain (semi-analytic) galaxy formation and evolution models and to compare them with the mean ages derived for intermediate and high redshift galaxies.

The method constructs, from color-color diagrams, cumulative age distributions based on a comparison with population synthesis isochrones. Cumulative age distributions are computed for the observed data, as well as for many Monte-Carlo simulations assuming two sub-populations of varying age and relative fraction. The observed and simulated distributions are compared, and the best model is assumed for the observed galaxy. Some details are given below.

\subsection{The observational basis: color-color diagrams}

The observational basis of the method are the optical-infrared color-color diagrams. They are shown in Fig. 1 for our eight sample galaxies. From these, it becomes clear that a direct photometric determination of the absolute ages of individual globular clusters is not possible.

However, the density distribution of objects in a diagram can be compared to the location of simple stellar population (SSP) models. In our analysis, we used models by Bruzual \& Charlot (2003); Vazdekis (1999); Maraston (2005) to probe the model dependence of our results. A visual inspection of Fig. 1 allows us to identify differences between the various galaxies: most systems have the majority of their clusters scattered around the oldest (here $13 \mathrm{Gyr}$ ) isochrone, while a few systems show a large fraction of clusters below that isochrone, i.e. hinting at younger ages.

\subsection{Quantifying the age structure: cumulative age distributions and best fitting model}

The procedure to quantify the age structure of a system is described in detail in Hempel et al. (2003) and Hempel \& Kissler-Patig (2004). Cumulative age distributions are derived with respect to SSP isochrones for our observed globular cluster systems (see Fig. 2) as well as for simulated systems of known properties (Fig. 3). The various simulations are based on a combination of two sub-populations, the older one having a fixed age of $13 \mathrm{Gyr}^{1}$ and the second varying in age between 1

$113 \mathrm{Gyr}$ is chosen given that the first generation of globular clusters is almost as old as the universe, for which we assumed an age as derived by the WMAP experiment (e.g. Spergel et al. 2003). 

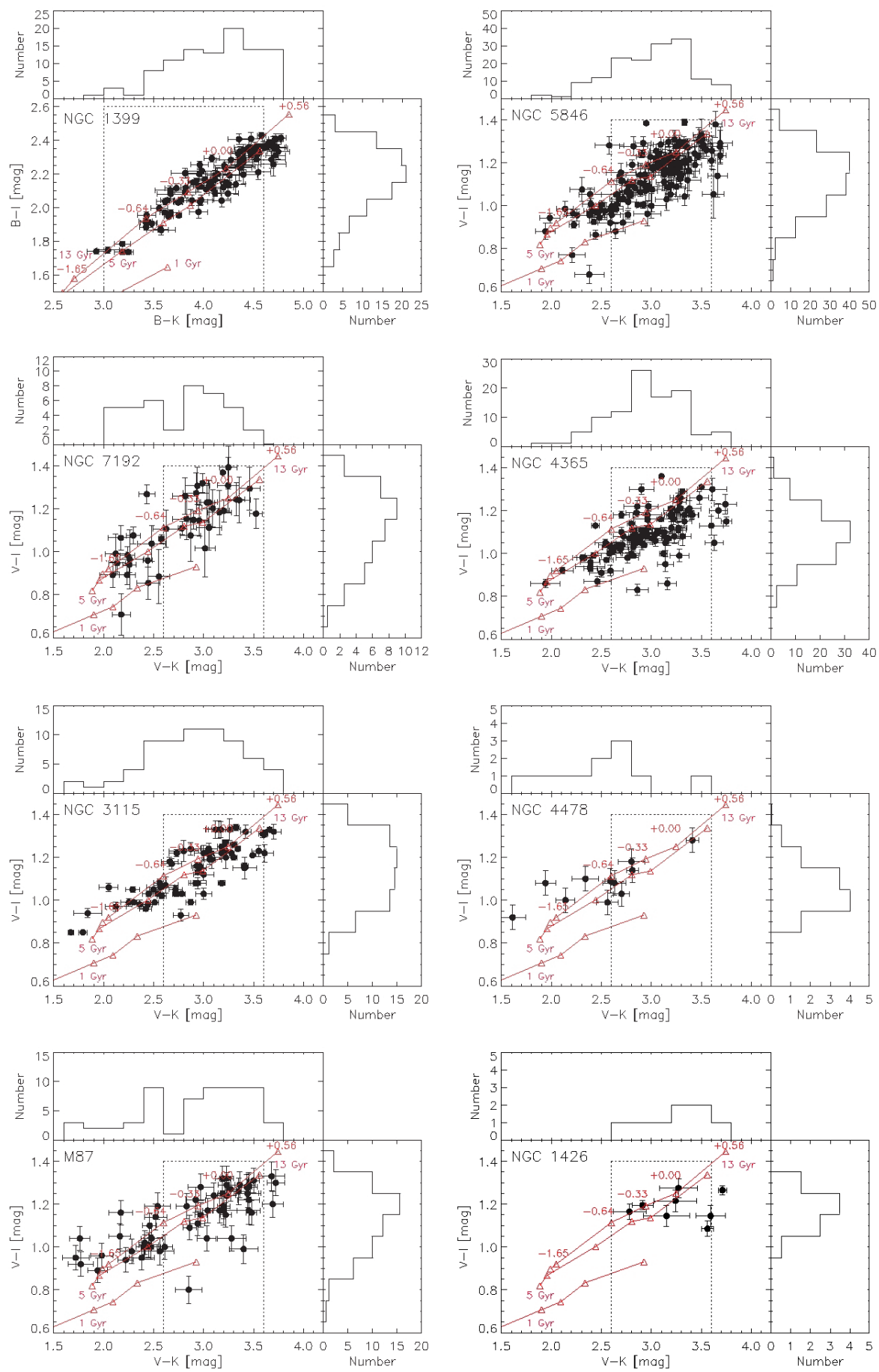

Fig. 1. Color-color diagrams and color distributions for the globular cluster systems included in this study. Only objects following the error cut $(\delta(V-I) \leq 0.15 \mathrm{mag}$ and $\delta(V-K) \leq 0.15)$, as used for further analysis, are plotted. The isochrones (marked in red) follow the SSP models by Bruzual \& Charlot (2003) and refer to a 1,5 and $13 \mathrm{Gyr}$ old stellar population. The metallicity increases with $(V-K)$ from -2.25 up to +0.56 , and is marked by triangles. Metallicity values are shown for the 13 Gyr isochrone.

and 13 Gyr. Simulations and observations are compared via a $\chi^{2}$-test and the simulation best fitting the observed age distribution is determined.

Note that we apply a color selection before building the cumulative age distribution in order to restrict the sample to metalrich globular cluster sub-populations (as well as to limit the contamination by foreground stars and/or background galaxies). The metal-rich globular clusters form the red sub-population in the near-infrared color distribution (e.g. $(B-K),(V-K))$ for ages $\geq 1$ Gyr. We assume that all metal-poor globular clusters are members of a first generation of globular clusters and do not consider them further. Also, the age resolution of our method, mostly driven by the optical color, decreases with metallicity (e.g. Fig. 1) and becomes impractical at the very metal-poor end of the distribution. The selection of metal-rich objects is based on limits to the near-infrared color, e.g. $(V-K)$ or $(B-K)$, respectively. We apply a color selection of $2.6 \leq(V-K) \leq 3.6$ or $3.0 \leq(B-K) \leq 4.6$, as indicated by the dashed lines in Fig. 1 .

Thus, our derived best fitting fraction of old to young globular clusters is computed for the metal-rich clusters only. 

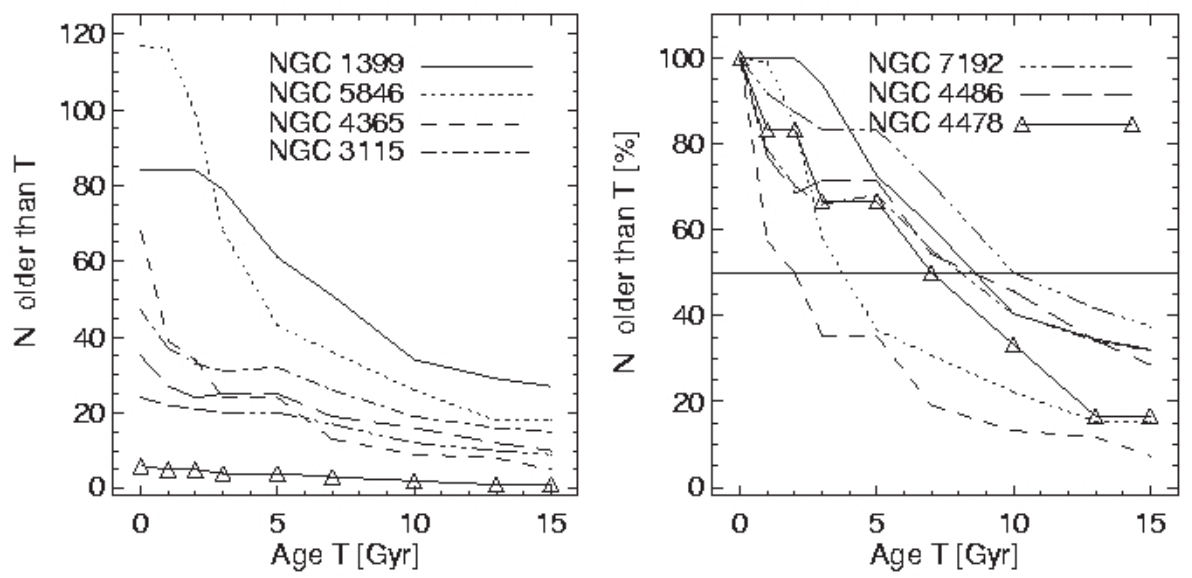

Fig. 2. Cumulative age distribution as derived for the selected globular cluster samples in various galaxies. The left panel shows the absolute number counts of objects older than a given isochrone T, which are normalized to the total number of objects included in the procedure (Hempel \& Kissler-Patig 2004). The SSP models used are by Bruzual \& Charlot (2003). For comparison we normalise the age distribution to the total number of objects included in the sample (see error cuts, color selection in Sect. 2.3). The normalised age distribution is shown in the right panel.

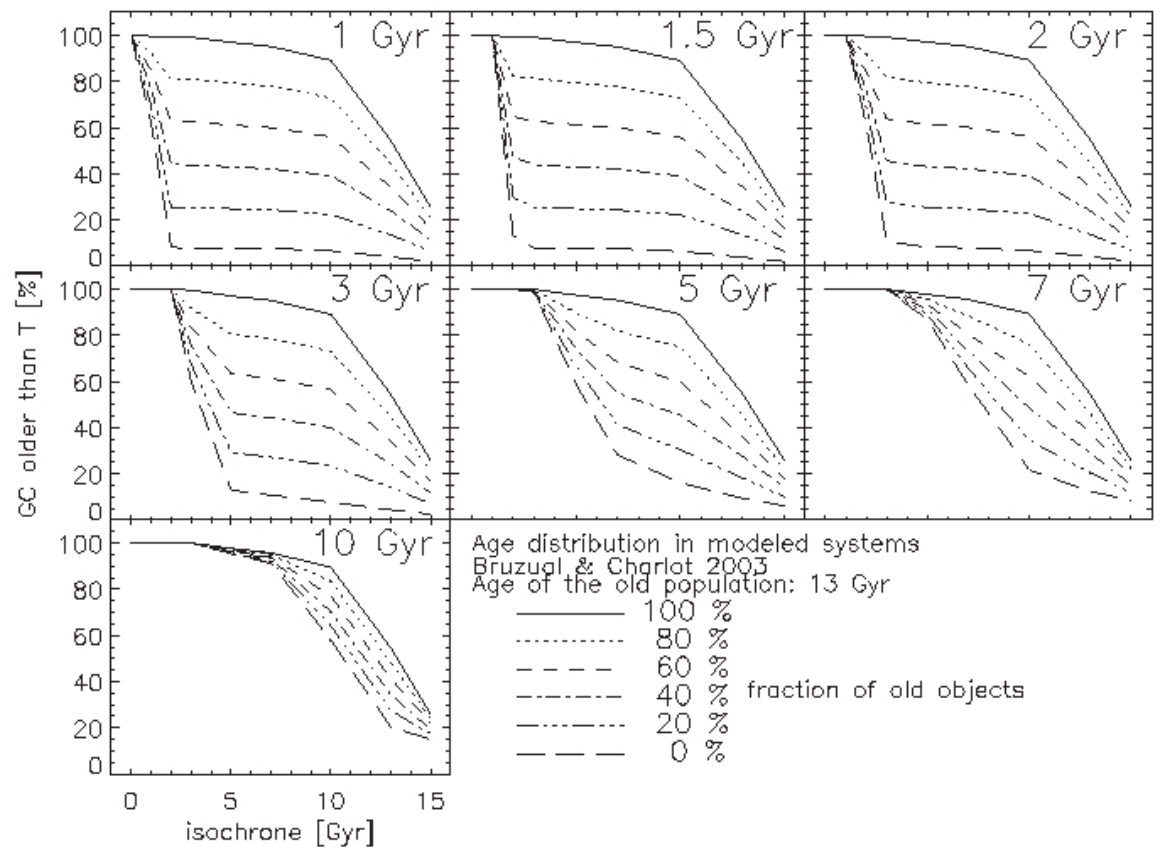

Fig. 3. Example for cumulative age distributions for simulated color distributions, based on the Bruzual \& Charlot SSP model (Bruzual \& Charlot 2003). Previous tests (Hempel \& Kissler-Patig 2004; Hempel et al. 2003) have shown that the choice of a specific SSP model does not affect the detection of globular cluster age sub-populations, but becomes important when the metallicity for each age population is derived (see Hempel et al. 2005).

To compute the total number of young clusters in a system, one also would have to consider the metal-poor population - typically as large as the entire metal-rich population. Further, our studies typically focus on a region within one effective radius, i.e. biases due to different radial distributions of the different sub-populations could also be present.

Photometric age estimates are based on SSP models, e.g. by Bruzual (2000), Bruzual \& Charlot (2003), Vazdekis (1999), or Maraston (2005). Our choice of the Bruzual \& Charlot models was originally based on the large age range provided by those models. Nevertheless, in Hempel \& Kissler-Patig (2004) we compare the results for the various SSP models mentioned above and conclude that, within the accuracy of the relative ages, the SSP model does not change the results significantly. However, new SSP models have become available (Maraston 2005), which now include a scatter in the HB structure at a given metallicity. These SSP models allow for two different HB morphologies and we now need to determine how strongly our results are affected by it. In Fig. 4 we plot the difference in both the $(V-I)$ and $(V-K)$ color between a "red" and "blue" HB morphology for various metallicities. Considering that in our study only metalrich $\mathrm{GCs}([\mathrm{Fe} / \mathrm{H}] \geq-0.35 \mathrm{dex},(V-K) \geq 2.6)$ are included, but only the colors for very metal-poor objects are affected by the HB morphology, we do not expect significant changes in our analysis. We also note that the color differences are well below the photometric uncertainties (error cuts $\leq 0.15 \mathrm{mag}$ ) and systematic uncertainties in the age estimates will therefore be dominated by the photometric uncertainties.

Before we present the results of our study and draw first conclusions on the age structure in the observed GCSs we 

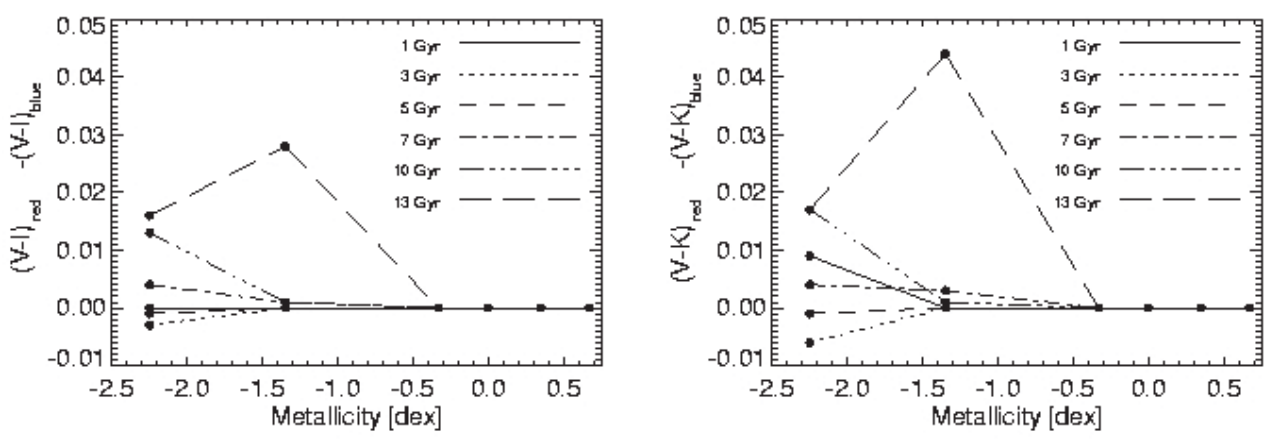

Fig. 4. $(V-I)$ and $(V-K)$ color differences in SSP model isochrones (Maraston 2005), assuming a different horizontal branch structure. Given that the color differences are well below the photometric accuracy and that only objects more metal-rich than $[\mathrm{Fe} / \mathrm{H}]=-0.35$ are included, the morphology of the horizontal branch does not affect our results.

emphasize briefly the limitations of the applied method. Details can be found in Hempel \& Kissler-Patig (2004).

- This method detects GC age sub-populations and sets constraints on their relative age and observed fractions. Individual GC ages are out of reach and require follow-up spectroscopy, if feasible.

- Besides a selection by photometric error $(<0.15 \mathrm{mag})$ we also select only metal-rich objects, given that for metal -poor GCs the age indicator, i.e. $(V-I)$ or $(B-I)$ color index, differs less than the error limit between a 13 Gyr and a 2 Gyr isochrone. Combined with the spatial bias, due to the limited size of the FOV, the observed ratio between the old and younger GC sub-populations is considered to be an upper limit. Deep wide-field studies will allow us to constrain this ratio more accurately.

- The cumulative age distribution in observed and simulated GCSs is derived as the number counts of GCs redder in ( $V-$ $I)$ or $(B-I)$ and hence older than a given model isochrone. First tests have shown that this requires a minimum number of objects to give statistically robust results (Hempel \& Kissler-Patig 2004). For $(V-I)$ vs. $(V-K)$ color distributions, with an error cut of $0.15 \mathrm{mag}$ we derive that $\sim 100 \mathrm{ob}-$ jects are required. The number of objects may be reduced for data sets with smaller errors, such as the WFPC2/NICMOS data set (Kundu et al. 2005).

- We compare the cumulative age distribution in the observed GCSs with simulated systems consisting of only two distinct age populations: one 13 Gyr old and a second, younger population. This is based on the assumption that a younger generation of globular clusters is formed during galaxy interactions, possible but still not very frequent events. To disentangle a larger number of age sub-populations will require a higher age resolution as well as a significantly larger number of objects.

\section{Resulting age distributions for our sample galaxies}

We present, and comment briefly on, the best fitting age structures of the individual systems of our sample. For this purpose, the galaxies have been sorted into cluster, group and isolated environment. As mentioned in Sect. 1 the galaxy environment is one of the galaxy parameters affecting the galaxy evolution.

\subsection{Cluster galaxies: NGC 1399, M 87 and NGC 4478}

NGC 1399 is a prime target for globular cluster research, displaying an extremely rich and extended globular cluster system. It has been the subject of numerous population studies using both photometry (e.g. Ostrov et al. 1993; Kissler-Patig et al. 1997; Dirsch et al. 2003) and spectroscopy (e.g. Grillmair et al. 1994; Kissler-Patig et al. 1998; Forbes et al. 2001; Richtler et al. 2004). Combined optical and near-infrared observations (HST/WFPC2, NICMOS3) have been presented by Kundu et al. (2005). Nevertheless, as described in Hempel \& Kissler-Patig (2004) the determination of the age structure via the cumulative age distributions and the successive comparison with simulated systems requires a significant number of globular clusters. We present in Appendix A new optical-infrared data for a significantly larger sample. The best fitting model is shown in Fig. 5. We find that $60-80 \%$ of the metal-rich population in our sample appears old, while the rest of the star clusters would have ages between one and a few Gyrs. Our results agree well with the results presented by Kundu et al. (2005), which in turn support previous spectroscopic work by Kissler-Patig et al. (1998) and Forbes et al. (2001).

Studies of the M 87 globular cluster system are manifold and led to controversial results. Based on Keck spectroscopy Cohen et al. (1998) found no sign of age differences between the globular clusters. Neither did Jordán et al. (2002), using Strömgren photometry obtained with HST/WFPC2. On the other hand, globular cluster sub-populations with an age difference of 3-6 Gyr were claimed by Kundu et al. (1999). The combined optical and near-infrared colors of the globular clusters used in this study and first published by Kissler-Patig et al. (2002) are mostly consistent with an old $(\approx 13 \mathrm{Gyr})$ population. Nevertheless, a small very red $((V-K)>3.4)$ sub-population is found that shows $(V-I)$ colors that are too blue for an old (10-13 Gyr) population. The interpretation of the age distribution for this sample (see Figs. 5 and 2) is somewhat less conclusive than the one for NGC 1399 (mostly due to the smaller number of objects), but is best interpreted along the same lines: the dominant metal-rich sub-population is old, but there is evidence for a small intermediate age population as well.

NGC 4478, the nearest neighbour of M 87, is a faint elliptical and was studied by Kissler-Patig et al. (2002). NGC 4478 does not appear to host metal-rich globular clusters at all. Further analysis with respect to the cumulative age distribution is hampered by this fact, as well as by the small number of detected globular clusters. Nevertheless, we apply our analysis to the 6 globular clusters in the sample: the results are shown in Fig. 5. 

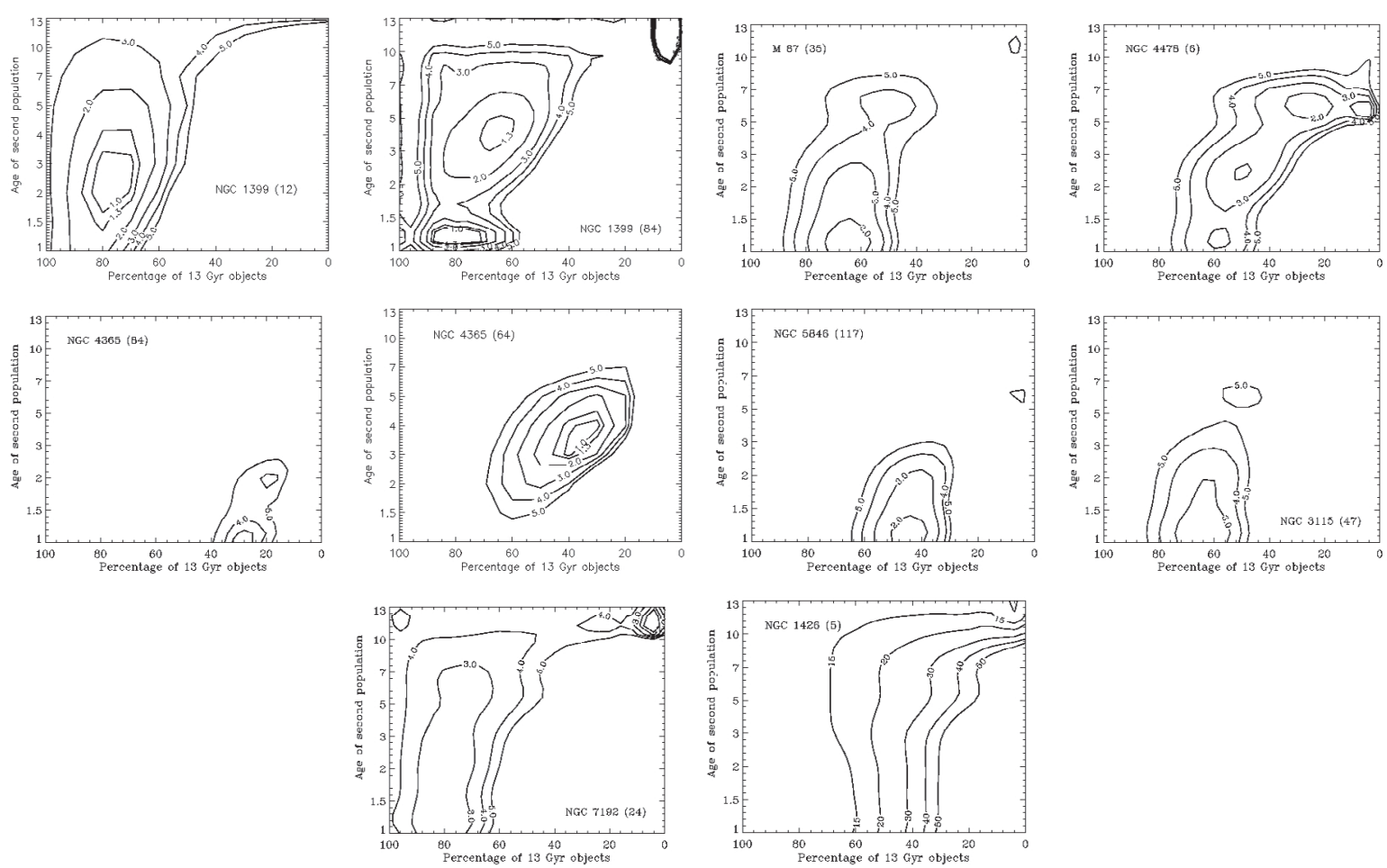

Fig. 5. $\chi^{2}$-contour plots for our sample galaxies, showing the best fitting models with respect to the age of the second population and the fraction of the (first) old population. The contour refer to the case in which the SSP models by Bruzual \& Charlot (2003) were used. The number of objects used for the fit is shown in parenthesis. For NGC 1399 the first panel shows the result based on WPFC2-NICMOS data (Kundu et al. 2005), for a sample of only 12 globular clusters, whereas the next panel represents the WFPC2-ISAAC sample with 84 globular clusters. The red population of NGC 1426 contains only 5 objects (after applying error and color cuts). The resulting age distribution suffers severely from low number statistics. For completeness the $\chi^{2}$-test was carried out as for the other galaxies in our sample, but taking the $\chi^{2}$-values into account we discard the globular cluster system of NGC 1426 from further discussion. For NGC 4365, the first panel shows the result based on HST- NICMOS data (Kundu et al. 2005), whereas the next panel represents the larger HST-ISAAC sample. For NGC 4478 (the close M 87 companion) the best fitting age/size combination is poorly constrained. M 87 is best fitted by a system dominated by an old stellar population, which is in agreement with Jordán et al. (2002). NGC 5846 is dominated by an intermediate age stellar population.

The result is, as expected, inconclusive, with multiple solutions equally favored. It serves as an example of how our method is affected by the sample size.

\subsection{Galaxies in (sub-)groups: NGC 4365 and NGC 5846}

Two galaxies in our sample, NGC 4365 and NGC 5846, dominate smaller groups of galaxies, or sub-groups in clusters. NGC 4365 hosts a kinematically- decoupled (counter-rotating) core (e.g. Surma \& Bender 1995; de Zeeuw et al. 2002). In the central region of NGC 5846, a prominent dust shell has been found (Goudfrooij \& Trinchieri 1998). In contrast to the X-ray faint galaxy NGC 4365, NGC 5846 shows a complex X-ray morphology, coupled with an equally peculiar energy distribution of the X-ray photons (Trinchieri \& Goudfrooij 2002).

The globular cluster system of NGC 4365 has attracted attention (Puzia et al. 2002; Hempel 2004; Brodie et al. 2005; Kundu et al. 2005), as it was the first globular cluster system claimed to show a significant intermediate age population based on the combination of optical and near-infrared photometry. In Fig. 5 we show the results of our $\chi^{2}$-test in two panels (one for the results purely based on HST (WFPC2/NICMOS3) data, Kundu et al. 2005; one for the results based on HST/WFPC2 and VLT/ISAAC data, Puzia et al. 2002). The good agreement between the results for both data sets gives strong support to our method. The age distribution in NGC 4365 is best represented by a composite of two equally important sub-populations: an old one and one of intermediate (few Gyr) age. While this interpretation, put forward in Puzia et al. (2002), Hempel \& Kissler-Patig (2004) and Kundu et al. (2005), is supported by the spectroscopic observations of Larsen et al. (2003), it is not supported by the ones of Brodie et al. (2005). A detailed discussion about the disagreement is given by Kundu et al. (2005).

Our second example of an early-type galaxy dominating a group environment is NGC 5846. The age structure of its globular cluster systems resembles the one found in NGC 4365: the metal-rich globular clusters in the inner effective radius appear to be best compatible with two equal sub-populations: an old and a young (few Gyr) one. The size and the relative ages of the two sub-populations differ as a function of the employed SSP models Hempel \& Kissler-Patig (see 2004, for details) but all suggest the combination of an old $(13 \mathrm{Gyr})$ and a significant young subpopulation in the metal-rich system.

\subsection{Isolated galaxies: NGC 3115, NGC 7192 and NGC 1426}

Our three isolated galaxies are accompanied only by dwarf galaxies (e.g. Puzia et al. 2000). These galaxies are unlikely to have been involved in major galaxy interactions inducing significant star formation. Indeed, NGC 7192 shows a very similar age structure as NGC 1399: there is no clear sign of a second, intermediate-age sub-population and the metal-rich system is compatible with being completely dominated by old clusters. NGC 3115 appears to have a similar age structure, although it 


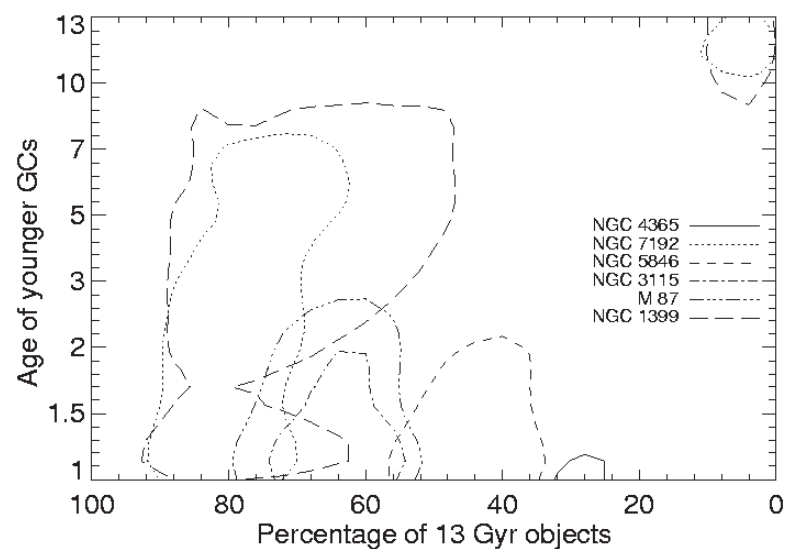

Fig. 6. $\chi^{2}$-contours for the globular cluster systems used in our study (with exception of NGC 1426 and NGC 4478). The contours indicate the best fits to the system's age distribution, based on the Bruzual \& Charlot (2003) SSP models.

may be closer to the one observed in M 87: a small fraction of the metal-rich globular clusters might be of younger ages. But the evidence for and size of this young population is much less pronounced as in NGC 4365 or NGC 5846. After applying the error and color selection in the NGC 1426 data, the remaining sample includes only 5 objects. As for NGC 4478 we show the formal result of our analysis in Fig. 5 but the small sample size makes the results inconclusive.

\section{Correlations between age structure and galaxy parameters}

In this section, we investigate the correlations between the age structures of our systems and the host galaxy properties. As already indicated in the previous section, we start by looking at trends with changing galaxy environment, before looking at trends with changing structural parameters. First, we define a parameter that quantifies the globular cluster system age.

\subsection{A metric for the age structure of a globular cluster system: the Methuselah parameter}

In order to quantify the age structure of a globular cluster system, we introduce the "Methuselah" parameter Mth, describing the ratio of old to young metal-rich globular clusters, weighted by age, as derived from the best fitting model for each data set (see Fig. 6) to the cumulative age distribution.

$\mathrm{Mth}=t_{\mathrm{pop} 1} * f_{\mathrm{pop} 1}+t_{\mathrm{pop} 2} * f_{\mathrm{pop} 2}$

where $t_{\text {pop } x}$ and $f_{\text {pop } x}$ refer to the age and fraction (respectively) of the two simulated populations. Note that the old population of globular clusters (pop1) is always assigned a fixed age of $13 \mathrm{Gyr}$. The age of the potential second population (pop2) as well as the fraction of each population is derived from the best fitting model. The parameter is derived for the metal-rich globular cluster population only. Note that a pure $13 \mathrm{Gyr}$ old population will have Mth $=13$, while e.g. a pure $5 \mathrm{Gyr}$ population will have Mth $=5$.

The Methuselah values for our sample galaxies are given in Table 2, as derived from the cumulative age distributions using the Bruzual \& Charlot (2003) models. With a significant intermediate age population in NGC 4365 and NGC 5846 both galaxies show the lowest Methuselah values, 4.6 (NGC 4365) and 5.8
Table 2. Methuselah values for our sample galaxies. The "old" GC population is assumed to be $13 \mathrm{Gyr}$ old, its relative size is derived as $f_{\text {pop } 1}=1-f_{\text {pop } 2}$.

\begin{tabular}{lcrrr}
\hline \hline Galaxy & $t_{\text {pop2 }}[\mathrm{Gyr}]$ & $f_{\text {pop2 }}$ & Mth & $\delta$ Mth \\
\hline NGC 1399 & 5 & 0.4 & 9.8 & 3.29 \\
NGC 3115 & 1 & 0.4 & 8.2 & 2.05 \\
NGC 4365 & 1 & 0.7 & 4.6 & 0.66 \\
NGC 4478 & 2 & 0.5 & 7.5 & 2.25 \\
M 87 & 1 & 0.4 & 8.2 & 1.64 \\
NGC 5846 & 1 & 0.6 & 5.8 & 1.28 \\
NGC 7192 & 1 & 0.2 & 10.6 & 5.30 \\
\hline
\end{tabular}

(NGC 5846). In comparison, for a globular cluster system dominated by an old population we derive Methuselah values of 9.8, 8.2 and 7.5 for NGC 1399, M 87 and NGC 4478, respectively. For the isolated galaxies we determine the Methuselah values of 10.6 for both NGC 7192 and NGC 1426, and 8.2 for NGC 3115.

The uncertainty in the Methuselah values is based on the uncertainty of the relative size of the young/intermediate age population chosen to be the size range for the contours with a $\chi^{2}$ value $\leq 3.0$. In the cases in which age and size of the young sub-population are degenerate (e.g. NGC 1399, NGC 7192), we use the $\chi^{2}$ contours for the dominating and in general old population.

\subsection{Age structure as a function of galaxy environment}

Understanding the age structure in globular cluster systems as a function of the environment can provide further evidence in favor of or against the competing galaxy formation scenarios (early star formation in monolithic collapse type scenarios (Larsen 1974, and later work), or star formation over a longer time scale, as in the hierarchical merging scenarios (White \& Rees 1978)). In "monolithic" scenarios, postulating an early collapse of all galaxies, we do not expect a strong environmental dependence. In contrast, in hierarchical scenarios, the star formation history is linked to the dissipational merger history, which in turn is a function of the environment.

We characterized the galaxy environment in two ways: qualitatively (deciding whether to call the galaxy environment "cluster", "group" or "isolated", see Sect. 3), and quantitatively, using the local galaxy density as derived by Tully \& Fisher (1988). The Tully density parameter reflects the number of galaxies brighter than $M_{\mathrm{B}}=-16$ per $\mathrm{Mpc}^{3}$ in the (todays) immediate environment of the galaxy. Both descriptions have advantages and disadvantages. Clearly, the former is arbitrary but allows us to include information that is hard to quantify (exact position in a group/cluster, infall velocity, X-ray halos); the latter parameter has the advantage of being objective.

In Sect. 3 we divided the galaxies in qualitative environments and showed that the two group galaxies both showed a significant fraction of intermediate-age, metal-rich globular clusters, i.e. underwent significant star formation episodes at later times than cluster or isolated galaxies.

In Fig. 7, we show the correlation of Mth with the quantitative environment parameter $\rho$. A weak trend is seen in the sense that Mth decreases with $\rho$, in other words galaxies in denser environment appear to have younger globular cluster systems. However, this remains a visual impression as formal correlation tests (Pearson, Spearman rank tests) only barely favor the hypothesis of a non-zero correlation. A correlation between 


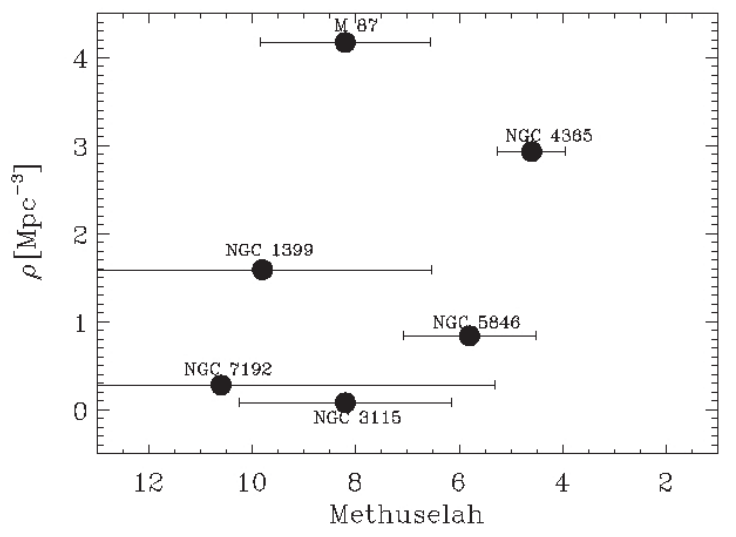

Fig. 7. Correlation of the Methuselah parameter Mth with the immediate galaxies environment, described by the Tully density $\rho$.

Mth and the galaxy environment is thus supported in our sample by the fact that we find significant intermediate- age subpopulations only, but in all group galaxies of our sample. The Mth against $\rho$ correlation does not reinforce this claim but is compatible with it.

\subsection{Age structure as a function of galaxy internal parameters}

Beyond the correlation of the globular cluster age structure with galaxy environment, we investigate any potential correlation with internal parameters of the host galaxy. We first look for correlations with galaxy size, as traced by the central velocity dispersion $\sigma$ and the absolute luminosity $M_{V}$, before turning to parameters tracing the diffuse light properties $\left(B_{V}\right.$ and $V-K$ integrated colors, as well as $\mathrm{H}_{\beta}$ Lick index).

\subsubsection{Correlation with galaxy size}

The Methuselah parameter is plotted against the galaxies' $\sigma$ and $M_{V}$ in Fig. 8. No correlation is apparent, as confirmed by Kolmogorov-Smirnov tests, and various correlation tests favor the no-correlation hypothesis.

The age structure of the globular cluster system does not seem to depend on the galaxy size.

\subsubsection{Correlation with integrated stellar light properties}

For completeness, we compare the age structure of the globular clusters with the properties of the integrated galaxy light. We used the globular clusters to track the age structure because of the aforementioned problems with the integrated light (such as age-metallicity degeneracy, luminosity weighted quantities, etc.). The expectations are unclear. The Methuselah parameter is plotted against integrated colors and Lick $\mathrm{H}_{\beta}$ index in Fig. 9.

Our formal correlation tests show no correlation of the Methuselah parameter with galaxy integrated color (visually, NGC 1399 with its very red integrated color, reduces any potential correlation). Yet, the tests show a weak preference $(80 \%)$ for a correlation (as opposed to no correlation) in the case of Methuselah against the $\mathrm{H}_{\beta}$-line index. The most straightforward interpretation of a high $\mathrm{H}_{\beta}$ index is a luminosity weighted average younger age (although cf. Maraston \& Thomas 2000, for a different interpretation in some cases). Our result would therefore support the presence of an intermediate aged globular
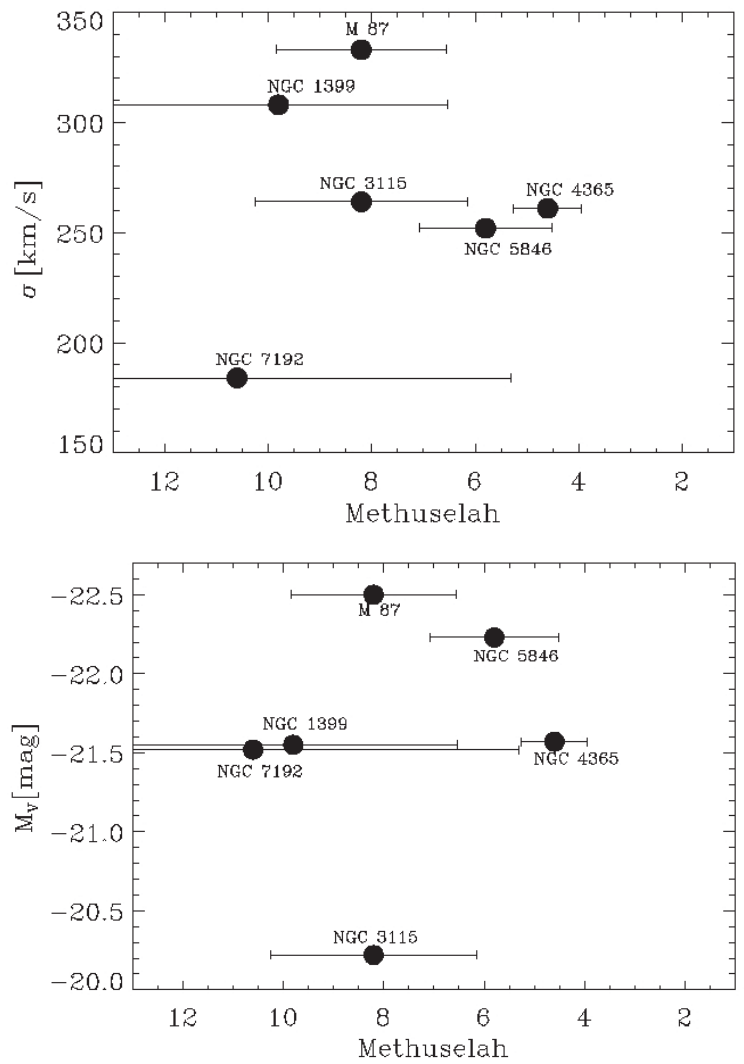

Fig. 8. The Methuselah parameter as a function of the mass-dependent central velocity dispersion $\sigma$ (top panel) and total $V$-band luminosity $M_{V}$ (bottom panel).

cluster population as inferred from the high Methuselah value. Thus, the sign of significant recent star formation activity as traced by the age structure of the globular cluster system could be supported by hints in the integrated light of the host galaxy.

This study includes eight globular cluster systems, some of which are too sparse to draw strong conclusions (e.g. NGC 1426, NGC 4478). When searching for correlations between the age structure, as represented by the Methuselah parameter, and various host galaxy parameters, our current galaxy sample is a first step towards probing the range of host galaxy parameters and needs to be extended in order to investigate the found tentative correlations in greater detail. The growing number of studies (Hempel et al. 2006, submitted) will allow us to constrain those correlations more tightly.

\section{Summary and conclusions}

We have presented the cumulative age distribution of the metalrich globular clusters of a sample of early-type galaxies. We compared these with simulated age distributions and determined the best fitting model under the assumption that the metal-rich globular clusters show two sub-populations: an old (13 Gyr) one and a younger one - their ratio being a fitting parameter. To quantify the age structure of the globular cluster system, we introduce the Methuselah parameter ( average age).

The investigated systems show a wide variety of ratios and ages of the younger sub-population (see Fig. 6). While most systems are dominated by an old metal-rich sub-population, a few show a dominating fraction of intermediate-age globular clusters, hinting at a significant epoch of recent star formation. 

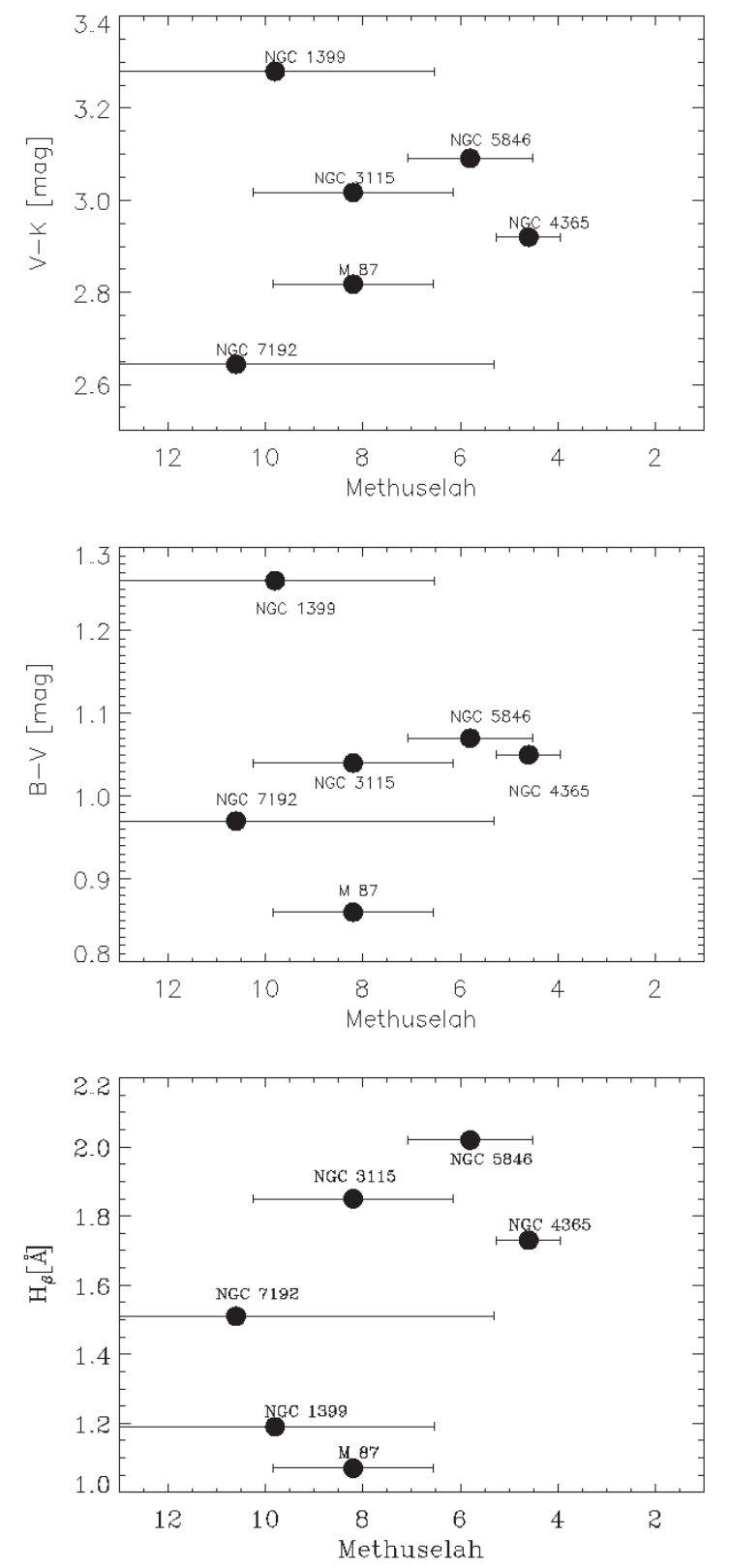

Fig. 9. The Methuselah parameter as a function of $(V-K)(t o p),(B-V)$ (middle) and the $\mathrm{H}_{\beta}$-line index (bottom), representing the stellar populations within the galaxy.

The clearest correlation between the age structure (the Methuselah parameter) and a galaxy property is with environment. The two galaxies in a group-type environment show the youngest Methuselah parameters, while the galaxies in clusters and in isolated environments are predominantly old. The age structure does not correlate with galaxy size, but appears to be weakly correlated with the $\mathrm{H}_{\beta}$ index of the host galaxy integrated light (in the sense that a lower Methuselah parameter is seen in galaxies with a young integrated light measurement).

We conclude that globular cluster systems are good tracers of recent star formation activities in early-type galaxies, and that group galaxies appear to form a significant fraction of their globular clusters/stars in the more recent past than do cluster of elliptical galaxies. Qualitatively similar conclusions were drawn from surveys of the integrated light of galaxies (e.g. Kauffmann et al. 2003a,b; Denicoló et al. 2005; Puzia et al. 2005).
Acknowledgements. We thank A. Kundu for providing the optical photometry on NGC 1399 prior to publication. T.H.P. acknowledges support by NASA through grant GO-10129 from the Space Telescope Science Institute, which is operated by AURA, Inc., under NASA Contract NAS5-26555. This research has made use of the NASA/IPAC Extragalactic Database (NED) which is operated by the JET Propulsion Laboratory, California Institute of Technology, under contract with the National Aeronautic and Space Administration. We also thank the anonymous referee for her/his comments which greatly helped to improve the manuscript.

\section{Appendix A: NGC 1399 and NGC 1426 observations and data reduction}

The optical data for both galaxies were obtained with HST/WFPC2, using the broad band filter F450W and F814W for NGC 1399 and $F 555 W$ and $F 814 W$ for NGC 1426 , respectively. The total exposure times were $5200 \mathrm{~s}$ in the $F 450 \mathrm{~W}$ for NGC 1399 and $1800 \mathrm{~s}$ in F555W for NGC 1426, the F814W exposure times are $1600 \mathrm{~s}$ for both galaxies. The instrumental magnitudes were converted into $B$ (NGC 1399), $V$ (NGC 1426) and $I$ (NGC 1399, NGC 1426) band magnitudes of the Johnson Cousin system following the procedure given by Holtzman et al. (1995). Details on the data reduction can be found in Kundu et al. (2005) for NGC 1399 and in Gebhardt \& Kissler-Patig (1999) for NGC 1426.

The near-infrared data for both galaxies were obtained with the Infrared Spectrometer And Array Camera (ISAAC) attached to the Unit telescope 1 of the European Southern Observatory in service mode (ESO program 64.N-0102) during the nights of October 24/25/26/28 and November 19/20 in 1999. For both targets the same observing strategy was applied: $2 \times(18 \times 10 \mathrm{~s})$ on source $+2 \times(6 \times 10 \mathrm{~s})$ on sky $+2 \times(18 \times 10 \mathrm{~s})$ on source. The field of view covers $2.5 \times 2.5$, the pixel scale is $0.147 /$ pixel. The standard star observations revealed that photometric conditions were not given during the total observing run but only during the nights of Oct. 26 and Nov. 21, hence allowing the calibration of 13 (NGC 1399) and 22 (NGC 1426) bright objects. We determined the photometric offset between the calibrated $K$-band magnitude of those objects and their instrumental magnitude in the combined master frame (mean offset of $26.265 \mathrm{mag}$ for NGC 1399 and $26.087 \mathrm{mag}$ for NGC 1426). By conducting a curve of growth analysis the aperture correction between a 10 pixel aperture (diameter), and an "infinite" (22 pixel) one was determined to be $0.30 \mathrm{mag}$ and $0.34 \mathrm{mag}$ for NGC 1399 and NGC 1426, respectively. The photometry was carried out using the Source Extractor by Bertin \& Arnouts (1996). The final results were reddening corrected using the reddening values by Cardelli et al. (1989).

Using the IRAF task tfinder,wcsctran and geomap the pixel coordinates of the globular clusters detected in the optical bands were transformed into ISAAC pixel coordinates and matched with the near-infrared source lists. Before applying any selection criteria such as color cuts or error limits, the two samples contained 145 and 31 objects in NGC 1399 and NGC 1426, respectively. The selection by error cut reduces the sample size for NGC 1399 to 99 and for NGC 1426 to 8. The later analysis followed Hempel \& Kissler-Patig (2004).

\section{References}

Bender, R., \& Renzini, A. 2005, Multiwavelength mapping of galaxy formation and evolution

Bender, R., \& Surma, P. 1992, A\&A, 258, 250

Bender, R., Burstein, D., \& Faber, S. M. 1992, ApJ, 399, 462

Bertin, E., \& Arnouts, S. 1996, A\&AS, 117, 393

Beuing, J., Bender, R., Mendes de Oliveira, C., Thomas, D., \& Maraston, C. 2002, A\&A, 395, 431 
Brodie, J. P., Strader, J., Denicoló, G., et al. 2005, AJ, 129, 2643

Bruzual, G. 2000, private communication

Bruzual, G., \& Charlot, S. 2003, MNRAS, 344, 1000

Cardelli, J. A., Clayton, G. C., \& Mathis, J. S. 1989, ApJ, 345, 245

Cohen, J. G., Blakeslee, J. P., \& Ryzhov, A. 1998, ApJ, 496, 808

de Vaucouleurs, G., de Vaucouleurs, A., Corwin, H. G., et al. 1991, Third Reference Catalogue of Bright Galaxies, Vol. 1-3, XII (Berlin, Heidelberg, New York: Springer-Verlag), 2069

de Zeeuw, P. T., Bureau, M., Emsellem, E., et al. 2002, MNRAS, 329, 513

Denicoló, G., Terlevich, R., Terlevich, E., Forbes, D. A., \& Terlevich, A. 2005, MNRAS, 358, 813

Dirsch, B., Richtler, T., Geisler, D., et al. 2003, AJ, 125, 1908

Djorgovski, S., \& Davis, M. 1987, ApJ, 313, 59

Dressler, A., Lynden-Bell, D., Burstein, D., et al. 1987, ApJ, 313, 42

Forbes, D. A., Beasley, M. A., Brodie, J. P., \& Kissler-Patig, M. 2001, ApJ, 563, L143

Gebhardt, K., \& Kissler-Patig, M. 1999, AJ, 118, 1526

Gorgas, J., Efstathiou, G., \& Salamanca, A. A. 1990, MNRAS, 245, 217

Goudfrooij, P., \& Trinchieri, G. 1998, A\&A, 330, 123

Goudfrooij, P., Alonso, M. V., Maraston, C., \& Minniti, D. 2001, MNRAS, 328 237

Grillmair, C. J., Freeman, K. C., Bicknell, G. V., et al. 1994, ApJ, 422, L9

Hempel, M. 2004, Ph.D. Thesis, Ludwig-Maximilians Universität, München, Germany

Hempel, M., Hilker, M., Kissler-Patig, M., et al. 2003, A\&A, 405, 487

Hempel, M., \& Kissler-Patig, M. 2004, A\&A, 419, 863

Hempel, M., Geisler, D., Hoard, D. W., \& Harris, W. E. 2005, A\&A, 439, 59

Hempel, M., Zepf, S., Kundu, A., Geisler, D., \& Maccorone, T. 2006, NearInfrared Observations of Globular Clusters in NGC 4472, NGC 4594, NGC 3585 and NGC 5813 and Implications for their Ages and Metallicities, submitted
Holtzman, J. A., Burrows, C. J., Casertano, S., et al. 1995, PASP, 107, 1065

Jordán, A., Côté, P., West, M. J., \& Marzke, R. O. 2002, ApJ, 576, L113

Kauffmann, G., Heckman, T. M., White, S. D. M., et al. 2003a, MNRAS, 341, 33

Kauffmann, G., Heckman, T. M., White, S. D. M., et al. 2003b, MNRAS, 341, 54

Kissler-Patig, M., Kohle, S., Hilker, M., et al. 1997, A\&A, 319, 470

Kissler-Patig, M., Brodie, J. P., Schroder, L. L., et al. 1998, AJ, 115, 105

Kissler-Patig, M., Brodie, J. P., \& Minniti, D. 2002, A\&A, 391, 441

Kundu, A., Whitmore, B. C., Sparks, W. B., et al. 1999, ApJ, 513, 733

Kundu, A., Zepf, S. E., Hempel, M., et al. 2005, ApJ, 634, L41

Larsen, S. S., Brodie, J. P., Beasley, M. A., et al. 2003, ApJ, 585, 767

Maraston, C. 2005, MNRAS, 362, 799

Maraston, C., \& Thomas, D. 2000, ApJ, 541, 126

Ostrov, P., Geisler, D., \& Forte, J. C. 1993, AJ, 105, 1762

Pahre, M. A. 1999a, in Star Formation in Early Type Galaxies, ASP Conf. Ser., 163,283

Pahre, M. A. 1999b, ApJS, 124, 127

Puzia, T. H., Kissler-Patig, M., Brodie, J. P., \& Schroder, L. L. 2000, AJ, 120, 777

Puzia, T. H., Kissler-Patig, M., Thomas, D., et al. 2005, A\&A, 439, 997

Puzia, T. H., Zepf, S. E., Kissler-Patig, M., et al. 2002, A\&A, 391, 453

Richtler, T., Dirsch, B., Gebhardt, K., et al. 2004, AJ, 127, 2094

Spergel, D. N., Verde, L., Peiris, H. V., et al. 2003, ApJS, 148, 175

Surma, P., \& Bender, R. 1995, A\&A, 298, 405

Trinchieri, G., \& Goudfrooij, P. 2002, A\&A, 386, 472

Tully, R. B., \& Fisher, J. R. 1988, Annales de Geophysique

Vazdekis, A. 1999, ApJ, 513, 224 